\title{
EL RESURGIMIENTO DE LA ECONOMÍA POLÍTICA EN LA CIENCIA POLÍTICA ACTUAL*
}

Aldo Madariaga

" DOI: https://doi.org/10.18601/01245996.v21n41.02. Recepción: 06-062017, modificación final: 08-04-2019, aceptación: 14-05-2019. Sugerencia de citación: Madariaga, A. (2019). El resurgimiento de la economía política en la ciencia política actual. Revista de Economía Institucional, 21(41), 21-50.

a Doctor en Economía y Ciencias Sociales. Profesor Asistente, Centro de Economía y Políticas Sociales, Universidad Mayor, Chile, e Investigador Adjunto, Centro de Estudios de Conflicto y Cohesión Social (COES), Chile (CONICYT/FONDAP/15130009), [aldo.madariaga@umayor.cl], [https:// orcid.org/0000-0002-1219-8355]. 


\section{El resurgimiento de la economía política en la ciencia política actual}

Resumen Este artículo describe el desarrollo histórico de las interpretaciones de la economía política, critica la pretensión hegemónica de la economía neoclásica y rescata la "nueva economía política": el estudio de la constitución social, política y moral de la economía. Muestra como se la concibe en la ciencia política actual y ofrece dos claves de análisis para orientar futuras investigaciones. Por último hace un recorrido por trabajos recientes en América Latina que recogen esta interpretación, en especial las revisiones de la literatura sobre 'economía política de la política económica', y algunos estudios sobre las variedades del capitalismo.

Palabras clave: economía política, ciencia política; JEL: A12, B25, B52

\section{The resurgence of political economy in today's political science}

Abstract In this article, we show the historical development of different conceptions of political economy, criticizing the hegemonic pretention of neoclassical economics, and highlighting what we call the "new political economy": the study of the social, political and moral constitution of the economy in history. We describe this interpretation's current disciplinary insertion in political science, and offer two analytical keys to orient future investigations. Finally, we survey recent works in Latin America that echo this interpretation, especially the revisions of the 'political economy of policy reform' literature and the widening of studies on varieties of capitalism.

Keywords: Political economy, political science; JEL: A12, B25, B52

\section{O ressurgimento da economia política na ciência política atual}

Resumo Este artigo descreve o desenvolvimento histórico das interpretações da economia política, critica a pretensão hegemônica da economia neoclássica e resgata a "nova economia política": o estudo da constituição social, política e moral da economia. Ele mostra como é concebida na ciência política atual e oferece duas chaves de análise para orientar pesquisas futuras. Por fim, ele faz um percorrido por obras recentes na América Latina que incluem essa interpretação, especialmente, as revisões da literatura sobre a "economia política da política econômica" e alguns estudos sobre as variedades do capitalismo.

Palavras-chaves: economia política, ciência política; JEL: A12, B25, B52 
H $\mathrm{n}$ los albores de las ciencias sociales, el estudio de las relaciones Centre la economía y la política, entre mercados y gobiernos, recibió el nombre genérico de "economía política". Desde finales del siglo XIX y durante buena parte del siglo XX, el desarrollo de la economía política se vio limitado por la creciente especialización dentro de las nuevas ciencias sociales y la consiguiente asignación del estudio de la economía a la ciencia económica. La hegemonía de la economía neoclásica en la disciplina y sus intentos de presentar sus herramientas como canon universal para las demás ciencias sociales contribuyeron a limitar aún más los análisis y el alcance de la economía política.

En contra de ello, en las últimas décadas se observa un resurgimiento de la economía política que la ha situado en un lugar privilegiado dentro de la ciencia política y ha llevado a lo que se puede llamar "nueva economía política". Rescatando la tradición de los clásicos, la economía política es un enfoque interdisciplinario y multi-método del estudio de la economía como ámbito en el que no solo confluyen consideraciones económicas, sino también sociales, políticas y culturales. Este artículo describe y analiza el resurgimiento de la economía política como subdisciplina de la ciencia política actual. Pretende oponer su definición y sus claves de análisis a las que proponen la economía neoclásica y sus variantes contemporáneas. Al hacer esto, presenta un panorama detallado de la investigación actual en economía política a través de las diversas claves analíticas, incluida la investigación sobre América Latina.

En un contexto global donde las transformaciones económicas tienen profundas consecuencias políticas, sociales y morales -así como ambientales-, donde las formulaciones tradicionales han perdido su capacidad explicativa $-y$ para qué decir predictiva- a la vez que es cada vez más claro que los elementos políticos, sociales y culturales son indisociables de los económicos, la visión de la economía política clásica y su resurgimiento en la ciencia política cobran aún más fuerza (Stilwell, 2016).

Este artículo intenta presentar el desarrollo histórico y actual de la economía política, y fijar una posición en los debates actuales en favor del pluralismo en economía política. En América Latina, busca fortalecer una visión alternativa a la que hoy es hegemónica. Desde la llegada de los Chicago Boys a Chile y su expansión en el resto del continente, la economía ha sido objeto de estudio exclusivo de los economistas y se ha intentado aislar al análisis económico del de otras disciplinas sociales. La visión de economía política, como hoy se interpreta en la ciencia política, liga de mejor manera el análisis 
de la economía a la democracia y el desarrollo, y permite entender cómo se pueden combinar los objetivos de eficiencia económica con otros tanto o más deseables, como el buen gobierno, la representación política, los derechos sociales y el cuidado del medio ambiente.

La primera sección hace un breve recorrido histórico para entender los significados asociados a la economía política. Tomando como referencia la tableau idéologique de Hirschman (1982), identifica cuatro significados actuales y sus características. Destaca la political economics, asociada a los intentos de la economía neoclásica por hegemonizar el estudio de lo político y lo social, y el rescate de la economía política clásica en la ciencia política actual. La segunda sección expone esta segunda visión, analiza cómo se ha convertido en subdisciplina de la ciencia política y describe sus principales claves de análisis. Estas claves se ordenan en dos ejes: uno referido a los factores explicativos (intereses, instituciones e ideas) y otro al nivel del análisis (doméstico o internacional). La tercera sección revisa el resurgimiento de la economía política así entendida en América Latina. La conclusión rescata los aportes de este resurgimiento para entender los desafíos que hoy enfrenta la región.

\section{¿QUÉ ES LA ECONOMÍA POLÍTICA? DE LA HEGEMONÍA NEOCLÁSICA A LA "NUEVA ECONOMÍA POLÍTICA”}

La definición de la economía política como el estudio de las determinaciones recíprocas entre economía y política resulta intuitiva, pero es insuficiente pues oculta un hecho fundamental: lo que se entiende por 'lo económico' y 'lo político' ha cambiado en la historia y en las mismas ciencias sociales (Caporaso y Levine, 1992; Clift, 2014; Milonakis y Fine, 2009; Weingast y Wittman, 2006; Chandhoke, 1994). Esta sección muestra que el cambio en la definición de la economía y su relación con la política ha dado origen a distintos enfoques. Se analiza especialmente la hegemonía a la que desde finales del siglo XIX aspira la ciencia económica, no solo en el estudio de la economía sino de los demás ámbitos de interacción social, incluida la política. Destaca, asimismo, los intentos actuales por rescatar la tradición histórica, que entendía la economía política como una intersección entre factores sociales, políticos y culturales que influyen en la economía y viceversa. Para ordenar estas definiciones se usa el tableau idéologique, elaborado por Albert Hirschman (1982) para ordenar las visiones existentes en ciencias sociales sobre la relación entre la economía de mercado y las relaciones sociales precapitalistas. 


\section{ECONOMÍA POLÍTICA EN LOS ALBORES DE LAS CIENCIAS SOCIALES}

El término economía proviene del griego oikos, la economía del hogar, la producción y distribución de bienes para satisfacer las necesidades de las unidades domésticas. Se empezó a denominar "economía política" alrededor del siglo xviı debido a una serie de transformaciones en la escala de la producción, la circulación y la distribución de bienes y servicios (Caporaso y Levine, 1992). Junto al surgimiento de los Estados nación en la Europa de la época se produjo una transformación gradual pero significativa en la manera de organizar la satisfacción de necesidades, trasladando el centro de operaciones del núcleo doméstico al aparato estatal. En este proceso fue fundamental la creación de mercados nacionales a medida que se iban organizando los nuevos Estados nación (Braudel, 1986; Heilbroner, 1974; Polanyi, 2011).

Estos mercados nacionales eran una innovación que difería de los mercados de ciudades-puertos para el comercio de larga distancia y de los mercados locales de comunidades pequeñas. Se comenzó a utilizar el adjetivo "política" para entender esta economía que, a diferencia de la anterior, vinculaba personas desconocidas (extraños, en vez de familiares o conocidos), y en la que la responsabilidad de satisfacer las necesidades recayó en una autoridad política, la cabeza del Estado, en vez de la cabeza del hogar (Caporaso y Levine, 1992).

Así entendida, en sus inicios la economía política era una disciplina orientada a aconsejar a los jefes de Estado acerca del manejo de los asuntos económicos para satisfacer las necesidades de los súbditos o de los nacientes ciudadanos (Caporaso y Levine, 1992). En ese contexto, se desarrolló en medio del debate sobre el rol del Estado en la economía y su responsabilidad para determinar y seleccionar qué necesidades se satisfacían y cómo satisfacerlas, siguiendo principios de buen gobierno. Gran parte de los economistas clásicos pertenecía a esta tradición, y hasta bien entrado el siglo xIx, se llamó economía política a la ciencia económica en general (Schumpeter, 1990, p. 57; Milonakis y Fine, 2009). En tiempos de los clásicos se acentuó la tendencia a autonomizar lo económico, ontológicamente-como ámbito diferenciado de la realidad, con sus propios principios y leyes-y epistemológicamente -como campo de estudio y manera de enfocar la realidad empírica (Dumont, 1982; Milonakis y Fine, 2009).

Este proceso, iniciado en el siglo XvII, guarda relación con una serie de procesos paralelos. En primer lugar, con la separación de lo económico y lo político, como muestran los trabajos de John Locke y sucesores (ver Macpherson, 1979). Estos autores pensaban que la propiedad y el intercambio provenían de un estado de naturaleza 
anterior a la sociedad civil, que implicaba consensos anteriores al contrato político, entre ellos el consenso sobre la convencionalidad del dinero y la obligatoriedad de los contratos comerciales. En segundo lugar, con la separación de lo económico y lo moral, en la medida que la búsqueda del interés egoísta no se consideraba perjudicial, sino que podía generar un orden social armónico. Mientras que antes la moral enseñaba que el egoísmo se debía subordinar a fines superiores, en la nueva ciencia económica, esta moralidad fue suplantada y obtuvo una cláusula de exclusividad: lo económico es el único ámbito donde es aceptable la búsqueda egoísta del interés, y donde, además, aumenta el bienestar de la población (Hirschman, 1999). Finalmente, se consolidó la idea de que el dominio de la economía es un todo coherente constituido por partes interrelacionadas que forman un sistema autocontenido de relaciones lógicas.

La revolución marginalista de finales del siglo XIX concluyó este largo proceso de autonomización de la economía y sentó las bases conceptuales y metodológicas de la nueva ciencia. Entonces se empezó a llamar economía (economics) al análisis de la economía propiamente dicha (economy), y economía política (political economy) para calificar peyorativamente los análisis - como el de la economía marxista- contaminados por consideraciones "no económicas", y por ello "politizadas", así como a un tipo de análisis pre-científico (Stilwell, 2016, p. 288). A esto contribuyeron los esfuerzos de pensadores como el sociólogo norteamericano Talcott Parsons, quien partiendo de la idea de que la especialización en ciencias sociales era un hecho moderno, impulsó la división del trabajo entre ellas y así legitimó el aislamiento de la economía (Streeck, 2012, p. 1; Milonakis y Fine, 2009, cap. 12).

\section{HACIA UN TABLEAU IDÉOLOGIQUE de LA ECONOMÍA (POLÍTICA)}

Hoy podemos identificar cuatro significados asociados al estudio de la economía política, cada uno vinculado a cierta manera histórica de entender la economía (Caporaso y Levine, 1992, pp. 21-32). Para ordenarlos, tomamos prestado de Hirschman su tableau idéologique (Hirschman, 1982) utilizando dos ejes: uno referido a la concepción ontológica de la economía como ámbito de la realidad, y el otro al carácter epistemológico de la economía como ciencia (cuadro 1).

En el cuadro se diferencia entre una definición restrictiva y una definición ampliada de la economía como ámbito de la realidad (dimensión ontológica), y entre una concepción interdisciplinaria de su estudio y una concepción autonomizada, siguiendo la división del trabajo entre disciplinas (dimensión epistemológica). 
Cuadro 1

Tableau idéologique de la economía política

\begin{tabular}{|c|c|c|c|}
\hline & & $\begin{array}{l}\text { Dimensión ef } \\
\text { economía }\end{array}$ & $\begin{array}{l}\text { istemológica: } \\
\text { omo ciencia }\end{array}$ \\
\hline & & $\begin{array}{c}\text { División } \\
\text { del trabajo }\end{array}$ & $\begin{array}{c}\text { Fertilización } \\
\text { cruzada }\end{array}$ \\
\hline $\begin{array}{l}\text { Dimensión } \\
\text { ontológica: } \\
\text { economía } \\
\text { como ámbito } \\
\text { de la realidad }\end{array}$ & $\begin{array}{l}\text { Definición restrictiva } \\
\text { Definición ampliada }\end{array}$ & $\begin{array}{l}\text { Enfoque tradicional } \\
\text { Enfoque residual }\end{array}$ & $\begin{array}{l}\text { Political economics } \\
\text { Nueva economía } \\
\text { política }\end{array}$ \\
\hline
\end{tabular}

Fuente: elaboración propia.

En el primer cuadrante se sitúa el "enfoque tradicional”, que concibe la economía como un ámbito diferenciado de lo social, donde se produce y circulan bienes para satisfacer necesidades. En la medida en que entiende que cada ámbito diferenciado de la realidad social opera con sus propias reglas y leyes invariantes, hay una división del trabajo entre ciencias sociales, de modo que a cada una le corresponde estudiar solo uno de esos ámbitos. En la economía (economy) la escasez es el principio de funcionamiento fundamental, y la economía (economics) es la disciplina que identifica las leyes invariantes que rigen dichos procesos (Polanyi, 2011; Heilbroner, 1974). En este enfoque, la economía política es la intervención del Estado en la economía, mediante sus regulaciones y la política económica, que se estudian con las herramientas de las distintas corrientes (Stilwell, 2016, p. 288).

La pax parsoniana, que mantuvo a cada ciencia social encerrada en su propia jurisdicción, fue rota abruptamente hacia la segunda mitad del siglo pasado. Entre los años cincuenta y setenta, autores asociados a las escuelas de Virginia y de Chicago emprendieron paralelamente un asalto explícito desde la ciencia económica a las demás ciencias sociales, pretendiendo lograr una hegemonía no solo sobre la economía como objeto de estudio sino sobre el conjunto de fenómenos sociales, políticos y culturales. Postularon la superioridad de las herramientas de la economía neoclásica, en particular la formalización y la matematización de sus postulados, destacaron la acumulación continua de evidencia a que conducen (Black, 1950) y publicaron trabajos influyentes como el de Anthony Downs sobre partidos políticos y comportamiento electoral, el de James Buchanan y Gordon Tullock sobre gobierno, reglas de decisión y diseños institucionales, y el de Mancur Olson sobre grupos de interés y acción colectiva (Stigler, 1984). 
Este proceso de colonización tuvo un gran impulso con la publicación, a principios de los setenta, de "El enfoque económico del comportamiento humano" de Gary Becker (1976). En este trabajo, Becker reclama la validez universal del modelo de elección racional y maximización de utilidades de la economía marginalista. Para Becker, este enfoque económico es aplicable a todo comportamiento humano, a todo tipo de decisiones, cualquiera que sea el motivo y cualquiera que sea el agente. Cuatro décadas después, Dani Rodrik, uno de los economistas críticos más respetados, destaca que "los modelos de los economistas son nuestra mejor guía cognitiva en las interminables colinas y valles que constituyen la experiencia social" (Rodrik, 2015, p. 8). Estos enfoques se sitúan en el segundo cuadrante.

En su intento de dar cuenta de las dinámicas propias de la política, los herederos de la economía neoclásica se refieren a la economía política como political economics (Persson y Tabellini, 2002), a la que definen como la aplicación del método y del análisis económico al estudio de la política (Weingast y Wittman, 2006). Una versión, con fuerte influencia de la economía neoclásica contemporánea, la nueva economía clásica -que supone expectativas racionales y se aplica al llamado ciclo económico-político (political business cycle) (Drazen, 2008; Fischer, 2008) - se ha ocupado en estudiar de qué modo la búsqueda del interés personal por los políticos (y las instituciones que la hacen posible)- limita el crecimiento económico. Ejemplo de ellos son los trabajos de los "Bocconi Boys", un grupo de economistas (A. Alesina, S. Ardagna, G. Tabellini, F. Giavazzi, etc.) que estudiaron economía en la elitista universidad Bocconi de Milán, asesoran a los organismos financieros internacionales ${ }^{1} \mathrm{y}$ han hecho carrera estudiando los factores políticos que impiden la adopción de políticas que su escuela considera deseables: bancos centrales independientes, austeridad fiscal, políticas monetarias rígidas (Alesina, Roubini y Cohen, 1997; Persson y Tabellini, 2002; Fischer, 2008) ${ }^{2}$.

Otro ejemplo es la aplicación del análisis neo-institucionalista al estudio del desarrollo, que subraya la importancia de las instituciones políticas para explicar el desarrollo de los países, usando una definición de las instituciones proveniente de la escuela de la elección pública y el método neoclásico (North, 2006; Acemoglu y Robinson, 2013). A

${ }^{1}$ En la reciente crisis europea, los "Bocconi boys" fueron el principal sostén intelectual de la austeridad fiscal impulsada por la troika formada por el FMI, la Comisión Europea y el Banco Central Europeo (Blyth, 2013). Sobre este grupo, ver Helgadóttir (2016).

2 Para una crítica basada en una teoría de las instituciones no neoclásica, ver Chang (2006). 
diferencia de la political economics más ortodoxa, para la cual-como planteó Hayek-la democracia liberal tiende a ser un obstáculo para el desarrollo (o la libertad), esta versión pregona que la democracia liberal asociada a la economía de mercado es el mejor arreglo institucional para lograr el desarrollo. Sus trabajos siguen la orientación neoclásica, como ilustra el tratamiento de uno de los fenómenos más estudiados por los politólogos -la transición a la democracia- utilizando la lógica y los métodos de esa escuela (Acemoglu y Robinson, 2006).

Una versión menos interesante desde el punto de vista analítico -aunque no menos influyente- parte de una definición ampliada de la economía, pero junto al enfoque tradicional defiende la división del trabajo entre ciencias sociales que se manifiesta en la imprecisa y unilateral concepción de la economía política (tercer cuadrante). A esta se la suele concebir como una situación de conflicto en la que hay ganancias materiales y actores con intereses distintos enfrentados. Así, por ejemplo, en un artículo titulado "La economía política del ébola", la periodista Leigh Phillips dice que "el ébola es un problema que no se revolverá porque resolverlo no es rentable"'3. A veces simplemente se analizan los aspectos, factores o consecuencias económicas de una actividad. Por ejemplo, en la Asociación Internacional de Investigación en Comunicaciones y Medios hay una sección de economía política que "examina el papel del poder en la producción, distribución e intercambio de la comunicación mediatizada [y] en la transformación de los mensajes en mercancías" (Wasko, 2013, pp. 5-6). En este caso, el análisis no se limita al campo económico - ni recurre al método neoclásico-; pero en principio podría haber "economía política" de cualquier cosa. Puesto que en esta versión hay una herencia disciplinar más laxa, no tiene un cuerpo teórico y metodológico sólido y no define de manera explícita qué entiende por economía, lo llamamos enfoque residual.

En el cuarto cuadrante se sitúa la "nueva economía política"4. Esta surgió en forma incipiente en la segunda mitad del siglo pasado, con la caracterización de la economía de posguerra como su etapa fordista. Esta corriente es heredera de esa fase específica, cuando se descubrió

3 [https://www.jacobinmag.com/2014/08/the-political-economy-of-ebola/]

${ }^{4}$ Se califica así para hacer un paralelo con el surgimiento de la nueva sociología económica en los años ochenta, que retomó la sociología económica clásica para superar la división del trabajo entre economía y sociología de posguerra, ver Smelser y Swedberg (2005). Siguiendo a Arrighi (2001), la principal diferencia entre ambas es el enfoque macro de la nueva economía política, que rescata elementos clásicos no recogidos por la nueva sociología económica, en particular el análisis del capitalismo y de su desarrollo.

Revista de Economía Institucional, vol. 2 I, N. ${ }^{\circ}$ 4I, Segundo semestre/20i9, Pp. 2 I-50 ISSN OI $24-5996 / \mathrm{E}-\mathrm{ISSN} 2346-245$ O 
la diversidad existente dentro de un mismo sistema económico, pues la exportación del capitalismo norteamericano a la Europa de posguerra dio lugar a formas institucionales distintas a las de Estados Unidos, que harían posible la existencia de un capitalismo eficiente pero socialmente integrador en los países europeos, y también en Japón (Streeck, 2018).

En esta tradición se pueden destacar los trabajos de la escuela francesa de la regulación sobre las diferencias institucionales entre el fordismo estadounidense y el francés (Aglietta, Boyer), los estudios de las instituciones de intermediación de intereses y los arreglos "neocorporativos" en Europa continental y su éxito para regular el conflicto entre capital y trabajo (Schmitter, Katzenstein), la literatura sobre sistemas sociales de producción que cuestionó la existencia de un modelo productivo único en el capitalismo avanzado (Piore y Sabel, Zeitlin, Streeck), así como los trabajos sobre los Estados de bienestar, los cuales encontraron que los distintos esquemas de política social eran resultado de la fortaleza relativa de los sindicatos en alianza con partidos de izquierda o socialcristianos, según el país (Korpi, Esping-A.). Cabe mencionar también la escuela estructuralista latinoamericana y las teorías de la dependencia, que cuestionaron la idea de una economía única asociada a la teoría del desarrollo de la época. Conceptos como "centro/periferia" de Raúl Prebisch, "desarrollo dependiente" de Cardoso y Faletto, y "estilos de desarrollo" de Aníbal Pinto, indicaban justamente que en la economía capitalista, incluso en las economías dependientes, existían diversas formas estructurales e institucionales y distintos actores sociales que of recían distintos caminos y posibilidades para el desarrollo económico (para una revisión, ver Palma, 1978).

Las lecciones de este cuerpo de literatura se condensaron en los años noventa, en lo que luego se conocería como la escuela de las "variedades del capitalismo" (Kitschelt, Lange, Marks y Stephens, 1999; Hall y Soskice, 2001), que intentó dar luces sobre las diversas maneras como los gobiernos democráticos pueden alcanzar los objetivos de eficiencia y crecimiento económico, y de justicia distributiva.

Este cuarto enfoque comparte con la antigua economía política su interdisciplinariedad, la inquietud por la relación entre Estado y mercado y el interés por integrar lo social y lo histórico en el análisis económico (Milonakis y Fine, 2009) y se diferencia de la tradición marginalista por su acento macro. Mientras que el enfoque económico beckeriano se basa en las herramientas de la microeconomía y la búsqueda de microfundamentos, la nueva economía política rescata 
la antigua economía política, en diálogo estrecho con otras corrientes, como la economía marxista, la macroeconomía keynesiana, la escuela histórica alemana y el institucionalismo norteamericano. En vista de que "la economía neoclásica se puede considerar como un desvío inicialmente interesante que se convirtió en un callejón sin salida (aunque con gran volumen de tráfico)" (Stilwell, 2016, p. 288), este enfoque abre un camino para salir de ese oscuro callejón.

\section{LA NUEVA ECONOMÍA POLÍTICA}

La nueva economía política concibe la economía como un ámbito históricamente situado. Aunque también estudia la producción, circulación y distribución de bienes y servicios, subraya que estos elementos no son invariantes, sino que toman formas históricamente definidas. Así, el énfasis recae en las instituciones y dinámicas políticas concretas que las sostienen en periodos específicos, lo que recupera su sentido original: estudiar la cambiante relación entre Estado y mercado o, tal vez mejor, entre capitalismo y democracia. En esto coincide con la tradición marxista, en la cual la economía y la política son analíticamente separables, pero ontológicamente imbricadas ${ }^{5}$. No es entonces solo disciplinariamente pluralista sino que deja de ser una subdisciplina con un tema limitado y se convierte en un campo de estudio amplio, cuyas fronteras se mueven a medida que la economía se transforma (Clift, 2014). El contrato y la propiedad privada, la búsqueda del interés personal y de la ganancia propios de las economías de mercado son producto de un conjunto específico de instituciones y dinámicas. La economía no es "economía" a secas, hoy es una economía capitalista, y en América Latina una economía dependiente. Para usar el término de Karl Polanyi, esta economía y este capitalismo están "empotrados" histórica y espacialmente (González y Madariaga, 2018).

Con respecto a lo social, la economía depende de estructuras de relaciones sociales y las reproduce. Buena parte de la producción y el intercambio económicos ocurren entre conocidos, parientes, amigos. En ese contexto, la confianza derivada de las estructuras de parentesco y de las instituciones que las favorecen puede ofrecer soluciones más efectivas que el mercado para garantizar los intercambios y el buen

${ }^{5}$ Los trabajos que caen dentro de esta definición han sido exhaustivamente discutidos e incorporados en la "nueva economía política". Una segunda variante marxista más ortodoxa, centrada en una exégesis de los trabajos de Marx más que en el rescate de la economía política clásica en su conjunto, no ha sido considerada en este análisis. 
desempeño económico (Hollingsworth y Boyer, 1997; Uzzi, 1997). Esta es una conclusión fundamental de los trabajos que estudian las ventajas del capitalismo alemán y japonés frente al anglosajón (Streeck, 2018). Por otra parte, la producción, el intercambio y la distribución se realizan en un espacio jurídico donde hay una autoridad o gobierno, y donde el interés individual está en tensión con el interés colectivo. En un régimen democrático, esa autoridad o gobierno no solo es responsable del cuidado de la economía, sino también de la sociedad (polity) y de la ciudadanía (Mair, 2015; Streeck, 2016). De modo que la economía está enlazada a la política, y la política económica no tiene que ver con la búsqueda de una solución óptima pues se dirime en el espacio político, donde no solo entran en juego consideraciones económicas o de eficiencia. Además, el ejercicio del poder político tiene consecuencias económicas y el ejercicio del poder económico tiene consecuencias políticas.

Por último, la economía tiene límites morales, o si se quiere, culturales: lo que se puede producir e intercambiar se define culturalmente (Sandel, 2013; Satz, 2010). Como es patente cuando bienes o servicios que históricamente estaban fuera del ámbito del mercado, como la educación o la seguridad social, se mercantilizan y transan como otras mercancías más. La expansión de la economía capitalista no solo es geográfica sino también moral (Sewell, 2008). En Chile, el país latinoamericano donde la mercantilización se ha impulsado con más fuerza, la re-politización de las políticas públicas - de la educación al medio ambiente pasando por la seguridad social-y el surgimiento de movimientos sociales que exigen replantear el papel del Estado intentan hacer efectivos esos límites e imponer fronteras políticas a la mercantilización en esos campos.

\section{NUEVA ECONOMÍA POLÍTICA Y CIENCIA POLÍTICA ACTUAL}

Debido a la exportación de la teoría de la elección racional a la ciencia política, la political economics ha ganado influencia en la manera de entender la economía política en la disciplina (Weingast y Wittman, 2006). No obstante, existe una corriente afín a la nueva economía política denominada "economía política comparada" (Clift, 2014). Esta sección presenta un panorama de la nueva economía política en ciencia política y algunas de sus claves analíticas para estudiar la economía.

La economía política comparada es una subdisciplina ecléctica en materia teórica y metodológica. En materia teórica tiene influencia de 
las principales ramas de la ciencia política, en especial de la política comparada, en su atención a las instituciones políticas; de las relaciones internacionales, en su interés por la economía transnacional y sus efectos locales; y de la política pública, en su análisis de las políticas que afectan la producción, el intercambio y la distribución: las políticas económicas propiamente dichas (fiscal, monetaria, industrial, comercial, etc.) la políticas sociales (educación, salud, pensiones, empleo), las políticas ambientales y energéticas. En materia metodológica privilegia la intersección entre métodos cuantitativos y cualitativos, y recurre a diseños comparados y a estudios de caso contextualizados mediante el rastreo de procesos (Pontusson, 2007).

También recibe el influjo de la sociología, en especial de la sociología económica (Smelser y Swedberg, 2005). Ejemplos de ello son el estudio de la construcción social y política de los mercados, de la sociología de las profesiones -cuando se analiza la influencia de los economistas y los tecnócratas en la política económica y los modelos de desarrollo- y de la importancia de los factores culturales en el proceso económico. Y, por supuesto, hay un fuerte influjo de la economía misma, pues pone a prueba sus hipótesis y utiliza sus herramientas. Algunos politólogos se han apropiado de la teoría económica para ofrecer una versión más realista -y empíricamente más plausible- de sus modelos. De acuerdo con Margaret Levi (2000, p. 830), gracias a ello la teoría económica ha recibido un gran aporte de los politólogos, especialmente en lo que atañe a la importancia de las instituciones y las normas, la riqueza del contexto, el estudio del conflicto y el poder, y la importancia de las motivaciones no egoístas.

Gráfica 1

Economía política en la ciencia política actual

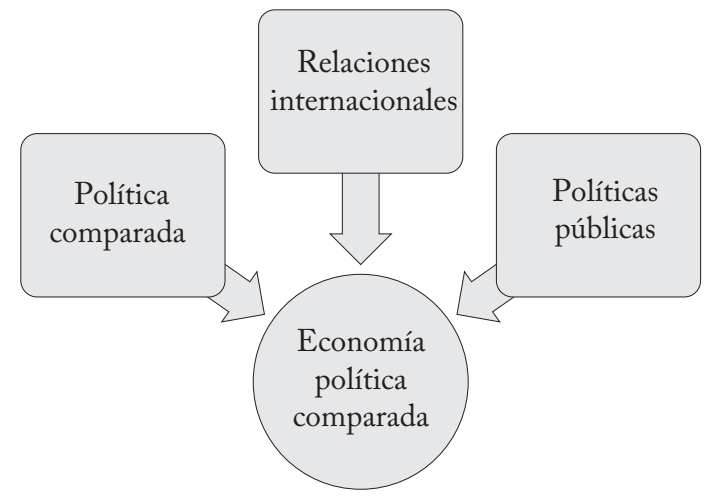

Fuente: elaboración propia. 
En la vasta producción de economía política en la ciencia política actual se distinguen dos claves de análisis. La primera, sobre los factores explicativos, y la segunda, sobre el nivel de análisis. Con respecto a la primera, Peter Hall (1997) argumenta que las explicaciones en economía política tienden a resaltar tres factores: los intereses, las instituciones y las ideas. Con respecto a la segunda, cabe distinguir entre economía política comparada, cuya unidad de análisis son los Estados-nación y sus relaciones, y la economía política internacional, cuya unidad de análisis es la interrelación entre el ámbito internacional y el doméstico.

\section{LAS TRES I: INTERESES, INSTITUCIONES E IDEAS}

Los intereses refieren a las condiciones materiales de vida y a los beneficios que proporcionan las distintas actividades. Los intereses determinarían las motivaciones, aspiraciones y metas de los agentes sociales, y también sus preferencias de política. Los intereses se suelen definir según la posición de los agentes en la estructura productiva y, más en general, en la estructura social. En opinión de Gourevitch, la estructura económica de un país da luces sobre "la situación de los actores sociales en la economía internacional, sus preferencias de política, sus bases potenciales para la alianza o el conflicto con otras fuerzas, y las coaliciones que surgen" $\left(1986\right.$, p. 59) ${ }^{6}$.

Cabe distinguir dos tipos de intereses: intereses económicos, que generan coaliciones de productores, e intereses políticos, que suelen llevar a coaliciones políticas (Hall, 1997) ${ }^{7}$. Hay dos maneras de determinar los intereses, una deductiva y otra inductiva. La más común es la deductiva, que infiere los intereses a partir de las consecuencias distributivas de las operaciones económicas o de políticas específicas. Para ello, se recurre a teorías que indican las divisiones relevantes entre la población o los posibles efectos distributivos se deducen directamente de las políticas. Se suelen emplear teorías estructuralistas que determinan los intereses según la relación de los agentes sociales con los medios de producción -que dividen la población en capitalistas, trabajadores y rentistas- o que determinan los intereses según clivajes políticos o culturales (centro-periferia, religioso-secular, etc.).

${ }^{6}$ Para críticas desde otras perspectivas, ver Geddes (1994), Schneider (2004) y Woll (2008).

7 En la vertiente marxista que remonta a autores como Antonio Gramsci y Nicos Poulantzas, el concepto de "bloques sociales" alude a coaliciones duraderas entre grupos económicos y políticos. 
También se pueden hacer subdivisiones dentro de los grandes clivajes. Por ejemplo, para Marx la principal división era entre capitalistas y proletarios, y entre los capitalistas diferenció a los industriales y los financieros. Eso también se puede hacer con los trabajadores: manuales, de servicios, "aristocracia obrera". Gourevitch (1986) y Frieden (1991) presentan criterios para distinguir intereses dentro de grandes agrupaciones. Estas subdivisiones agrupan intereses diversos que pueden llevar a la formación de coaliciones inter o poli-clasistas de grandes consecuencias políticas. Por ejemplo, América Latina se debate sobre el carácter nacional o transnacional del empresariado, sus preferencias de política y las consecuencias para la formación de coaliciones progresistas entre empresarios y trabajadores (BresserPereira, 2011; O'Donnell, 1978). Cabe destacar el trabajo de Eduardo Silva (1996), que analizó el cambio en las bases económicas de apoyo al régimen militar chileno, de sectores empresariales internacionalizados con activos líquidos (finanzas) a sectores internacionalizados con activos fijos (exportadores), que llevó de una fase ortodoxa a una fase pragmática en la aplicación del modelo neoliberal.

En la perspectiva del individualismo metodológico - muy común en la political economics-, los intereses se asocian al carácter racional y egoísta de los individuos, y su esfuerzo por maximizar sus beneficios. Así, los políticos buscan maximizar los votos - para llegar o mantenerse en cargos de elección popular-y los votantes, maximizar los beneficios esperados de votar por uno u otro candidato. Con respecto a esta relación recíproca, y la posibilidad de que los gobernantes manipulen la economía para aumentar los beneficios de los votantes y con ello la posibilidad de ser reelegidos, se han escritos trabajos sobre lo que se conoce como ciclo económico-político (Franzese, 2002; Drazen, 2008).

La manera inductiva para determinar los intereses, menos utilizada porque es intensiva en material empírico, consiste en utilizar encuestas de opinión y estudios de estratificación social para identificar las preferencias distributivas (Amable, 2009; Berens, 2015).

La segunda clave analítica se refiere a las instituciones. En economía política se las entiende como reglas que estructuran la actividad económica, estableciendo lo que se puede y no se puede hacer, y sancionando el incumplimiento. Buena parte de la investigación en economía política se nutre de la economía neo-institucionalista, en la cual las instituciones son un quinto factor (junto con el capital, la tierra, el trabajo y la tecnología) del desempeño económico (Ayala, 1999): las instituciones establecen incentivos, amplían el horizonte 
de las transacciones económicas, completan los mercados y reducen los costos de transacción.

En contraste con esta interpretación, desde una óptica más sociológica Wolfgang Streeck (1997) concibe las instituciones como "limitaciones favorables": mejoran el desempeño económico no porque impulsen la búsqueda de ganancias sino porque la controlan, y limitan la tendencia autodestructiva del capitalismo al fomentar la cooperación ${ }^{8}$. Algunos estudiosos de las instituciones de negociación colectiva en Europa continental argumentan que la institucionalización del poder sindical reduce la posibilidad de que las empresas bajen los salarios y despidan trabajadores, lo que fomenta la cooperación y las estrategias de mercado que compiten por calidad y no por precio, con mayores niveles de capacitación y calificación de la mano de obra, mejor distribución del ingreso, menores niveles de conflicto laboral y mejor adaptación a crisis externas (Katzenstein, 1985; Hall y Soskice, 2001).

Una segunda manera de ver la importancia de las instituciones es considerarlas como reglas del juego político, que asignan recursos y establecen incentivos y limitaciones a los actores políticos. En las teorías sobre recursos de poder, las instituciones laborales (negociación colectiva, co-determinación, etc.) aumentan el poder de negociación de los sindicatos y contribuyen a la creación de Estados de bienestar de corte socialdemócrata (Korpi, 1983; Esping, 1990). Garret y Lange (1996, p. 53) presentan una lista de instituciones políticas que se utilizan como variables independientes en los análisis de economía política: sistema político (autocrático vs. democrático, presidencial vs. parlamentario), leyes electorales (proporcionales vs. mayoritarias), agencias independientes como las cortes constitucionales y bancos centrales independientes. Se suele utilizar el término "actores de veto" para referirse a estas instituciones políticas. Aquí cabe mencionar los trabajos de Levitsky y Murillo (2010, 2013), quienes argumentan que en América Latina las instituciones tienen distintos niveles de cristalización y cumplimiento, y que por tanto es necesario analizarlas junto con los intereses y el poder de los agentes sociales para entender los frecuentes cambios institucionales en los países de la región?.

${ }^{8}$ Esta definición difiere de la que se asocia a la Political economics, según la cual las instituciones limitan el poder discrecional de los gobiernos y su interferencia en la economía (ver Rodrik y Zeckhauser, 1988).

9 Para una crítica de los análisis institucionalistas que no consideran el poder y los intereses de los actores que las sostienen, ver Pontusson (1995), Amable y Palombarini (2009) y Thelen (2012).

Revista de Economía Institucional, vol. 2 I, N. ${ }^{\circ}$ 4I, Segundo semestre/2oi9, Pp. 2 I-50 ISSN OI 24-5996/E-ISSN 2346-2450 
Una innovación es el concepto de "complementariedades institucionales", es decir, que las instituciones que determinan el desempeño económico forman un todo y generan sinergias, positivas o negativas (Hall y Soskice, 2001). Hall y Franzese (1998) discuten la creencia de que un banco central autónomo siempre logra menor inflación y mayor crecimiento. En vez de examinar únicamente el efecto de la independencia del banco central, analizan la interacción entre bancos centrales independientes y negociación colectiva, y su efecto conjunto sobre la inflación y el crecimiento. Estudian cuatro combinaciones (con y sin banco central independiente, con y sin negociación colectiva) y encuentran que la combinación de alta independencia del banco y alto nivel de negociación colectiva es la que da el mejor resultado de alto crecimiento y baja inflación. El concepto de complementariedades institucionales se extendió a los análisis de las "variedades del capitalismo" para incluir diversas instituciones (negociación colectiva, relaciones industriales, sistema financiero, sistema educativo y formación de competencias, política social, etc.) que generan sinergias (Hall y Gingerich, 2009). La idea central es que no se puede pensar en reformas institucionales o de política pública en forma aislada, y que es necesario entender cómo afectan a las instituciones y políticas complementarias $^{10}$.

Por último, en las dos últimas décadas han aparecido trabajos que muestran que las ideas son esenciales en el estudio de la política comparada y de la economía política (Garcé, 2015; Schmidt, 2008). Esos trabajos conciben las ideas como orientaciones normativas o cognitivas que inciden en la manera como los agentes identifican e interpretan sus intereses, e indican estrategias para alcanzarlos. En este tipo de análisis se considera que lo importante para entender la acción política no son los (supuestos) intereses objetivos de los actores ni las instituciones que enmarcan sus opciones sino cómo perciben esos intereses e instituciones, pues esas percepciones son la base de sus estrategias. En suma, las ideas constituyen marcos para entender el mundo e inciden en el comportamiento de los actores definiendo la clase de acciones que les parecen satisfactorias o legítimas.

Existen diferentes tipos de ideas: creencias, valores e ideologías; paradigmas de política y conocimiento formalizado en teorías. En economía política comparada se utilizan como factores explicativos, en especial en los trabajos que explican el cambio de las políticas económicas. Entre esos trabajos cabe destacar los que hacen énfasis

10 Para una crítica del carácter funcionalista de estas explicaciones, ver Streeck (2018).

Revista de Economía Institucional, vol. 2 i, N. ${ }^{\circ}$ 4I, Segundo semestre/2oig, Pp. 2 i-5o ISSN OI $24-5996 / \mathrm{E}-$ ISSN $2346-245^{\circ}$ 
en el aprendizaje, los cuales consideran que la política económica varía según se consolidan y agotan ciertos "paradigmas", como el paso del keynesianismo al monetarismo ${ }^{11}$; los que subrayan el papel de las comunidades epistémicas, por ejemplo, el papel de los economistas en las reformas de mercado en América Latina (Domínguez, 1996; Montecinos y Markoff, 2016), y los que destacan la difusión de las ideas y su valor cognitivo -en el sentido de que ofrecen soluciones prácticas-, por ejemplo, para explicar la difusión de las reformas de los sistemas de pensiones en América Latina (Weyland, 2007).

\section{ECONOMÍA POLÍTICA COMPARADA Y ECONOMÍA POLÍTICA INTERNACIONAL}

Un resultado central en el estudio de la economía política en las últimas décadas ha sido la constatación de la importancia de los factores internacionales (Stallings, 1992). Los trabajos sobre el tema, que destacan la interrelación entre las unidades nacionales y el efecto sobre su autonomía, así como las dinámicas globales, constituyen una rama de estudios que se conoce como economía política internacional y tiene influencia directa de las teorías sobre relaciones internacionales.

Se suele considerar que la economía política internacional es una disciplina con temas de investigación y referentes propios (Cohen, 2008; Gourevitch, 1996). Ese sería el caso de trabajos como los de Wallerstein y Cox sobre la relación entre el desarrollo del capitalismo, la distribución del poder y la dominación internacional, o el de Gilpin sobre la globalización. No obstante, cabe recordar la definición de Frieden y Martin (2002), para quienes la economía política internacional cubre todos los trabajos que tratan los elementos internacionales como variables dependientes o independientes; una definición que sugiere una interacción entre economía política comparada y economía política internacional que deseamos destacar. La gráfica 2 muestra la relación entre ambas subdisciplinas considerando si en sus análisis los factores domésticos o internacionales se toman como variables dependientes o independientes ${ }^{12}$.

${ }^{11}$ El estudio del surgimiento y los cambios en las familias de ideas, cómo se institucionalizan y se vuelven dominantes se ha consolidado luego de los trabajos pioneros de Peter Hall (1993). Dos estudios recientes son el de Mirowski y Plehwe (2009) sobre el nacimiento y la difusión de las ideas neoliberales, y el de Blyth (2013) sobre el ascenso de la idea de "austeridad" como principio de la política económica.

${ }^{12}$ La distinción entre intereses, instituciones e ideas como variables explicativas se replica a nivel doméstico e internacional. 
Gráfica 2

Formas de explicación en economía política internacional

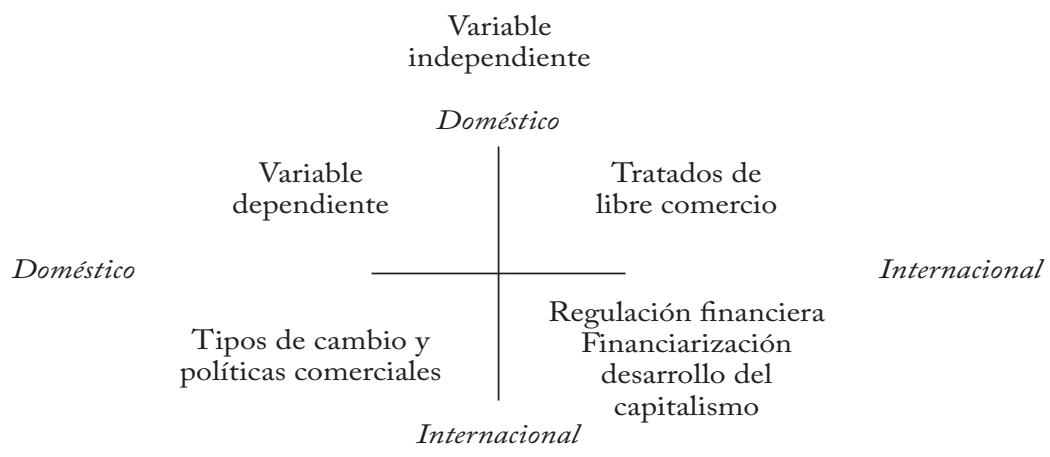

Fuente: elaboración propia.

En primer lugar, tenemos los estudios donde la variable dependiente es el ámbito doméstico y la independiente el ámbito internacional, como los que examinan los efectos de la economía internacional en las preferencias y cambios de preferencias de política de los agentes domésticos. Por ejemplo, Broz y Frieden (2006) muestran que la exposición a la competencia internacional determina las preferencias en materia de regímenes y niveles de tasas de cambio; y Kingston (2001), que el proceso de liberalización a nivel mundial modificó las preferencias de los industriales brasileños, que pasaron de preferir la protección y la intervención del Estado a preferir la apertura y la liberalización comercial. Otros autores muestran que la financiarización de la economía internacional, y organizaciones internacionales como el FMI y la OMC, limitan el margen de las políticas locales (Stallings, 1992; Kaplan, 2013; Campello, 2015).

En segundo lugar, tenemos los estudios donde la variable dependiente es el ámbito internacional y la variable independiente el ámbito doméstico, como los trabajos sobre tratados de libre comercio. Por ejemplo, Mansfield y Milner (2012) argumentan que la participación en acuerdos comerciales preferenciales -y la influencia de los Estados en su diseño- depende del tipo de régimen político (autoritario o democrático) del país y del número de actores de veto existentes. Wehner (2011) y Bull (2008) argumentan que, en el caso de Chile, los intereses de los sectores económicos más dinámicos de la economía, y la existencia de una red de técnicos en el gobierno, explican por qué en los años noventa Chile buscó asociarse con los países desarrollados (Nafta y Unión Europea) y no con bloques regionales como el Mercosur. 
Por último, tenemos los estudios en los que el ámbito internacional es una variable dependiente e independiente, como los trabajos que estudian interacciones a nivel internacional; por ejemplo, el de Kastner (2014) sobre el efecto de los movimientos sociales de consumidores en la regulación financiera después de la crisis de 2008. Así como los trabajos sobre dinámicas internacionales que prescinden de una categorización formal entre variables dependiente e independiente, por ejemplo, los que estudian procesos a gran escala, que cubre al mismo tiempo la economía internacional y los Estados nación. Dos de los fenómenos más estudiados son el proceso de financiarización iniciado en los años ochenta (Strange, 1998; Krippner, 2012) y las transformaciones del capitalismo y su relación con la democracia liberal (Mair, 2015; Sewell, 2008; Streeck, 2016; Wallerstein, Collins, Mann, Derluguian y Calhoun, 2013). Cabe mencionar también los trabajos sobre la crisis global, sus causas y sus efectos sociopolíticos (Bartels y Bermeo, 2013; Bermeo y Pontusson, 2012; Kahler y Lake, 2013), y sobre la preservación de la hegemonía de las corrientes neoclásica y neoliberal a pesar de la crisis (Blyth, 2013; Crouch, 2011; Mirowski, 2013; Schmidt y Thatcher, 2013).

Analíticamente, la distinción propuesta para identificar el nivel de análisis del explanans y el explanandum se presta para hacer una sociología de la ciencia distinguiendo las corrientes existentes en el estudio de un mismo tema, como la economía política del desarrollo (ver Gourevitch, 1996). En un polo se podrían situar los trabajos sobre el capitalismo dependiente latinoamericano, en especial los de autores como Gunder Frank, Dos Santos y Marini, para quienes la estructura económica internacional -en particular la división internacional del trabajo- era freno para el desarrollo de los países de la periferia. E1 "desarrollo del subdesarrollo" replicaba las estructuras de dominación externas en el ámbito local. Así, todo el peso de la explicación del desarrollo nacional recaía en el ámbito internacional. En el polo opuesto se podrían situar las teorías de inspiración neoclásica, que ponen todo el peso de la explicación en los factores domésticos. El ámbito internacional solo of rece oportunidades que cada país debería aprovechar. La clave aquí es la capacidad del Estado para dotarse de las instituciones más favorables, como plantean North o Acemoglu y Robinson.

Entre estos dos polos se situarían los trabajos que equilibran ambas explicaciones, como los de la Cepal, que consideraban posible un "desarrollo dependiente" y destacaban la capacidad de los actores nacionales para hacer alianzas progresistas, en ciertas condiciones, Así, 
dada la influencia externa, el ámbito doméstico no solo es una variable dependiente sino que explica las diferencias de desarrollo entre países dependientes. Por su parte, la literatura sobre desarrollo tardío tributaria de los trabajos de Gerschenkron (en especial los estudios de países asiáticos de Johnson, Amsden, Wade y Haggard) plantea que la economía internacional impone límites a la vez que abre posibilidades. Así, el ámbito internacional no es una variable explicativa sino una variable interviniente: define el nivel de tecnología de los países desarrollados e impone requerimientos estructurales al desarrollo de los demás países. Pero el peso de la explicación del desarrollo recae en el ámbito doméstico, donde la estructura institucional, la capacidad del Estado y el tipo de coaliciones explican el nivel de desarrollo de estos países.

\section{LA NUEVA ECONOMÍA POLÍTICA EN AMÉRICA LATINA}

En América Latina hay una larga tradición de estudios de economía política, que en el siglo pasado se concentraron en el problema del desarrollo, como muestran los debates sobre la dependencia y las tesis de la escuela estructuralista. Con el auge de la political economics y las teorías de la elección racional, la tradición estructuralista, más macro, fue cuestionada fuertemente, y se intentó centrar los estudios en los intereses egoístas de los gobernantes y la incapacidad de las instituciones para obligarlos a adoptar las políticas más deseables (Geddes, 1994). En 2014, varios autores suscribieron un manifiesto que denunciaba la excesiva importancia que se atribuía a las acciones individuales, a la teoría de la elección racional y a los métodos estadísticos, y llamaba a revalorizar la economía política clásica en la región (Smith et al., 2014). Este manifiesto apareció en un momento de revitalización de la disciplina, que impulsó nuevos temas de estudio, avances teóricos y metodológicos. Parte de este impulso se plasmó en la creación de la red de economía política América Latina (Repal).

Entre las contribuciones de la última década se destacan dos tendencias: la revitalización de la "economía política de la política económica", que estuvo en auge en los años ochenta y noventa durante el clímax del consenso de Washington, y la incorporación de la región a la agenda de estudios sobre la diversidad del capitalismo contemporáneo y sus cambios.

La literatura sobre "economía política de la política económica" surgió en los años ochenta vinculada a los esfuerzos por salir de la década perdida. Poco a poco se entendió que la incapacidad o tardanza 
para adoptar el decálogo del Consenso de Washington tenía que ver con factores políticos e institucionales, y no con el buen o mal diseño de las políticas (Haggard y Kaufman, 1992; Nelson, 1990). Esa literatura tenía un sesgo neoclásico, pues tendía a considerar los factores políticos únicamente como facilitador u obstáculo para implementar la política económica, cuya bondad no se discutía ${ }^{13}$. Se planteó incluso que la mejor manera de avanzar en las reformas era usar fórmulas institucionales para aislar a los reformadores de las posibles presiones políticas o sociales. En la última década, esa literatura ha sido revisada y enriquecida con trabajos que dan mayor acento a la diversidad de experiencias, arreglos institucionales y coaliciones posibles ${ }^{14}$.

Aquí se destaca el trabajo de Etchemendy (2012), centrado en el ámbito doméstico, que examina las combinaciones entre instituciones políticas y coaliciones que llevaron a distintas trayectorias de liberalización, y al surgimiento de dinámicas sociopolíticas que influyeron en la sostenibilidad de las reformas de mercado. Cabe mencionar también los trabajos de Kaplan (2013) y Campello (2015) que, partiendo del ámbito internacional, estudian los factores que restringen el margen de maniobra de los gobiernos en materia de política económica. Estos se centran en las condiciones impuestas por la liberalización financiera y los mercados de bonos a nivel global, y la capacidad de los políticos locales para llevar o no a cabo sus programas electorales en ese contexto. Desde otra óptica, Weyland (2009) y Kaufman (2011) analizan la apertura del margen de maniobra causada por el boom de materias primas de la primera década de este siglo, la cual hizo posible que los gobiernos de izquierda de esos años tuvieran menos restricciones estructurales. Por su parte, desde una perspectiva institucionalista, Flores-M. (2012) analiza los factores que incidieron en que los gobiernos de izquierda adoptaran programas moderados o radicales. Destaca la relación entre partidos y Congreso, y la estructura partidaria en la composición de esos gobiernos, más moderada o más radical, según cuán institucionalizada fuera la elección de candidatos dentro de los partidos y cuán estrecha fuese la relación entre los partidos y el Congreso.

Se deben mencionar, además, los trabajos de Fairfield (2010,2015), que analizan el poder del empresariado en las democracias latinoamericanas. Un poder que es estructural o instrumental, según limite

${ }^{13}$ Para una crítica, ver Schamis (2002).

${ }^{14}$ Algunos autores no incluidos en esta revisión, pero cuyas contribuciones en los años noventa y dos mil fueron esenciales para la revitalización de la última década, incluyen a María V. Murillo, Andrew Schrank, Marcus Kurtz y Héctor Schamis. 
la agenda de los gobiernos -mediante amenazas de fuga de capital o desinversión- o se participe directamente en el diseño de las políticas. No se pueden omitir su trabajo teórico y metodológico sobre el concepto de poder empresarial y su análisis empírico de las reformas tributarias en tres países de la región (Fairfield, 2015, 2018). Otras contribuciones recientes han revivido la discusión sobre el papel de los tecnócratas en la política económica, que mucho influyeron en las reformas asociadas al Consenso de Washington (Domínguez, 1996; Montecinos y Markoff, 2016). Dargent (2014) estudia el diseño de la política económica en Colombia y Perú, y encuentra que los tecnócratas no solo actúan sin atender al sentir de la ciudadanía, sino muchas veces con autonomía de los gobiernos y de los empresarios.

Con respecto a la diversidad del capitalismo, hay antecedentes en los trabajos de autores de la Cepal y la escuela de la dependencia, pero durante muchos años los autores latinoamericanos no han participado en los debates de este fructífero programa de investigación. No obstante, en la última década, Schneider y sus colaboradores (Schneider, 2013; Schneider y Karcher, 2010; Schneider y Soskice, 2009; Madariaga, 2018) han impulsado el estudio de las "variedades del capitalismo" en la región. Schneider argumenta que los países latinoamericanos forman parte de un tipo de capitalismo "jerárquico”, en el que ni los mercados ni la cooperación determinan las relaciones económicas (a diferencia de los países anglosajones y de Europa continental) sino las relaciones de jerarquía: grandes grupos económicos diversificados y empresas transnacionales vs. pequeñas y medianas empresas; empresas con grandes ventajas regulatorias y fuerte ascendencia en los gobiernos vs. trabajadores en mercados de trabajo precarizados y flexibles.

Aunque recibió fuertes críticas, entre otras cosas por agrupar a estos países en un solo modelo de capitalismo, la propuesta de Schneider tiene méritos. Ante todo, como él mismo señala, la caracterización de la región como un capitalismo jerárquico resalta los aspectos que persisten más allá de las diferencias entre países y modelos de desarrollo, como el poder del empresariado y de las empresas transnacionales, la precariedad de los trabajadores y de su calificación y la gran desigualdad. Además, abre sendas analíticas que no han sido exploradas en la región (Madariaga, 2018). Por ejemplo, en materia de política social se ha estudiado el papel de los partidos políticos de izquierda y los sindicatos (Huber y Stephens, 2012; Pribble, 2013), pero no se ha abordado en forma expresa el papel de los empresarios para facilitar o bloquear ciertas formas de protección social. Así mismo, pese a su 
importancia para el desarrollo, aún no se ha estudiado el papel del tipo de educación que se imparte en estos países así como el de la formación de capital humano (Bogliaccini y Madariaga, 2017). La investigación sobre la diversidad del capitalismo ayudaría a entender las complementariedades entre mercado laboral, protección social y sistema educativo, y a salir de la trampa de "bajos ingresos, baja productividad, alta desigualdad" (Schneider, 2013).

\section{COMENTARIOS FINALES}

Durante mucho tiempo la economía política ha estado sometida a la presión de la teoría hegemónica para unificar el estudio de la economía y la política bajo la lógica del comportamiento egoísta, la elección racional, la maximización de utilidades y la búsqueda de la eficiencia. Este artículo describe la evolución y las características de la nueva economía política, una interpretación alternativa asentada en la ciencia política, y presenta un panorama de la investigación actual.

La nueva economía política concibe la economía como un ámbito situado históricamente, que no se puede entender prescindiendo de su interrelación con factores sociales, políticos y culturales. Es un vasto campo disciplinario, con alta producción teórica y empírica. A la variedad de interpretaciones se suma la variedad de aproximaciones disciplinarias y entradas analíticas. En un momento en que la economía y la política parecen estar en un punto de inflexión en todo el mundo, en el que las fronteras entre ambas parecen más porosas que nunca, los análisis de economía política que adoptan estas premisas son muy necesarios.

Es conveniente que las nuevas generaciones de científicos sociales hagan eco de esta literatura y mejoren la comprensión de la economía, los problemas del desarrollo y los déficits de la democracia en la región. Así darán nuevas luces sobre la capacidad de los Estados para perseguir objetivos como la justicia social y la sostenibilidad ambiental, tan legítimos y necesarios como la eficiencia económica.

\section{REFERENCIAS BIBLIOGRÁFICAS}

Acemoglu, D. y Robinson, J. A. (2006). Economic origins of dictatorship and democracy. Nueva York: Cambridge University Press.

Acemoglu, D. y Robinson, J. A. (2013). Por qué fracasan los paises. México DF: Crítica.

Alesina, A. Roubini, N. et al. (1997). Political cycles and the macroeconomy. Cambridge: The MIT Press. 
Amable, B. (2009). The differentiation of social demands in Europe. The social basis of the European models of capitalism. Social Indicators Research, 91(3), 391-426.

Amable, B. y Palombarini, S. (2009). A neorealist approach to institutional change and the diversity of capitalism. Socio-Economic Review, 7(1), 123-143.

Arrighi, G. (2001). Braudel, capitalism, and the New Economic Sociology. Review, 24(1), 107-123.

Ayala, E. J. (1999). Instituciones y economía: una introducción al neoinstitucionalismo económico. México DF: Fondo de Cultura Económica.

Bartels, L. y Bermeo, N. (2013). Mass politics in tough times: Opinions, votes and protest in the Great Recession. Nueva York: Oxford University Press.

Becker, G. S. (1976). The economic approach to human behavior. Chicago IL: University of Chicago Press.

Berens, S. (2015). Preferences on redistribution in fragmented labor markets in Latin America and the Caribbean. Journal of Politics in Latin America, 7(3), 117-156.

Bermeo, N. y Pontusson, J. (2012). Coping with crisis. Government reactions to the great recession. Nueva York: Russell Sage Foundation.

Black, D. (1950). The unity of political and economic science. Economic Journal, 60(239), 506-514.

Blyth, M. (2013). Austerity: The history of a dangerous idea. Nueva York: Oxford University Press.

Bogliaccini, J. A. y Madariaga, A. (2017). Skills regimes in Latin America: Education, inequality and economic specialization in comparative perspective. Presentado en la 5. ${ }^{a}$ conferencia anual de la Repal, Lima.

Braudel, F. (1986). La dinámica del capitalismo. México DF: Fondo de Cultura Económica.

Bresser-Pereira, L. C. (2011). From the national-bourgeoisie to the dependency interpretation of Latin America. Latin American Perspectives, 38(3), 40-58.

Broz, L. y Frieden, J. A. (2006). The political economy of exchange rates. En B. R. Weingast y D. A. Wittman (eds.), The Oxford handbook of political economy (pp. 587-598). Nueva York: Oxford University Press.

Bull, B. (2008). Policy networks and business participation in free trade negotiations in Chile. Journal of Latin American Studies, 40(02), 195-224.

Campello, D. (2015). The politics of market discipline in Latin America. Nueva York: Cambridge University Press.

Caporaso, J. A. y Levine, D. P. (1992). Theories of political economy. Nueva York: Cambridge University Press.

Chang, H. J. (2006). La relación entre las instituciones y el desarrollo económico. Problemas teóricos claves. Revista de Economía Institucional, 8(14), 125-136.

Clift, B. (2014). Comparative political economy: States, markets and global capitalism. Basingstoke: Palgrave Macmillan.

Cohen, B. J. (2008). International political economy: An intellectual history. Princeton, NJ: Princeton University Press. 
Crouch, C. (2011). The strange non-death of Neo-liberalism. Cambridge: Polity Press.

Dargent, E. (2014). Technocracy and democracy in Latin America: The experts running government. Nueva York: Cambridge University Press.

Domínguez, J. I. (1996). Technopols: Freeing politics and markets in Latin America in the 1990s. University Park, PA: The Pennsylvania State University Press.

Drazen A. (2008). Political business cycles. En Palgrave Macmillan (eds.), The new Palgrave dictionary of economics (pp. 109-154). Londres: Palgrave Macmillan.

Dumont, L. (1982). Homo aequalis. Génesis y apogeo de la ideología económica. Madrid: Editorial Taurus.

Esping-A., G. (1990). The three worlds of welfare capitalism. Princeton NJ: Princeton University Press.

Etchemendy, S. (2012). Models of economic liberalization. Business, workers, and compensation in Latin America, Spain, and Portugal. Nueva York: Cambridge University Press.

Fairfield, T. (2018). Poder estructural en economía política comparada: perspectivas desde la formulación de políticas públicas en América Latina. En F. González y A. Madariaga (eds.), La constitución social, politica y moral de la economía chilena (pp. 147-184). Santiago de Chile: RIL Editores.

Fairfield, T. (2010). Business power and tax reform: Taxing income and profits in Chile and Argentina. Latin American Politics and Society, 52(2), 37-71.

Fairfield, T. (2015). Private wealth and public revenue in Latin America: Business power and tax politics. Nueva York: Cambridge University Press.

Fischer, S. (2008) New classical macroeconomics. En: Palgrave Macmillan (eds.), The new Palgrave dictionary of economics (pp. 361-362). Londres: Palgrave Macmillan.

Flores-M., G. A. (2012). After Neoliberalism? The Left and economic reforms in Latin America. Nueva York: Oxford University Press.

Franzese, R. J. (2002). Electoral and partisan cycles in rconomic policies and outcomes. Annual Review of Political Science, 5(1), 369-421.

Frieden, J. A. (1991). Invested interests: the politics of national economic policies in a world of global finance. International Organization, 45(04), 425-451.

Frieden, J. A. y Martin, L. L. (2002). International political economy: Global and domestic interactions. En I. Katznelson y H. V. Milner (eds.), Political science. The state of the discipline (pp. 693-721). Nueva York: W.W. Norton.

Garcé, A. (2015). El institucionalismo discursivo como oportunidad La ciencia política latinoamericana y el estado del arte en la literatura sobre el poder político de las ideas. Politica y Gobierno, 22(1), 199-226.

Garrett, G. y Lange, P. (1996). Internationalization, institutions and political change. En R. Keohane y H. Milner (eds.), Internationalization and Domestic Politics (pp. 48-77). Cambridge y Nueva York: Cambridge University Press. 
Geddes, B. (1994). Challenging the conventional wisdom. Journal of Democracy, 5(4), 104-118.

González, F. y Madariaga, A. (2018). Introducción: des-economizar los mercados, socializar la economía. En F. González y A. Madariaga (eds.), La constitución social, politica y moral de la economía chilena (pp. 9-40). Santiago de Chile: RIL Editores.

Gourevitch, P. A. (1986). Politics in hard times: Comparative responses to international economic crises. Ithaca NY: Cornell University Press.

Gourevitch, P. (1996). La "segunda imagen invertida": orígenes internacionales de las políticas domésticas. Zona abierta, 74, 21-68.

Haggard, S. y Kaufman, R. R. (1992). The politics of economic adjustment. Princeton NJ: Princeton University Press.

Hall, P. A. (1993). Policy paradigms, social learning, and the state: The case of economic policymaking in Britain. Comparative Politics, 25(3), 275-296.

Hall, P. A. (1997). The role of interests, institutions, and ideas in the comparative political economy of industrialized nations. En M. Luchbach y A. Zuckerman (eds.), Comparative politics. Rationality, culture, and structure (pp. 174-207). Cambridge: Cambridge University Press.

Hall, P. A. y Franzese, R. J. (1998). Mixed signals: Central Bank independence, coordinated wage bargaining, and European Monetary Union. International Organization, 52(3), 505-535.

Hall, P. A. y Gingerich, D. W. (2009). Varieties of capitalism and institutional complementarities in the political economy: An empirical analysis. British Journal of Political Science, 39(03), 449-482.

Hall, P. A. y Soskice, D. (2001). Varieties of capitalism: The institutional foundations of comparative advantage. Nueva York: Oxford University Press.

Heilbroner, R. L. (1974). La formación de la sociedad económica. $3^{\mathrm{a}}$ ed. México DF: Fondo de Cultura Económica.

Helgadóttir, O. (2016). The Bocconi boys go to Brussels: Italian economic ideas, professional networks and European austerity. Journal of European Public Policy, 23(3), 392-409.

Hirschman, A. O. (1982). Rival interpretations of market society: Civilizing, Destructive, or feeble? Journal of Economic Literature, 20(4), 1463-1484.

Hirschman, A. O. (1999). Las pasiones y los intereses: Argumentos políticos en favor del capital. Barcelona: Peninsula.

Hollingsworth, J. R. y Boyer, R. (1997). Contemporary capitalism: The embeddedness of institutions. Cambridge: Cambridge University Press.

Huber, E. y Stephens, J. D. (2012). Democracy and the Left: Social Policy and Inequality in Latin America. Chicago IL: University of Chicago Press.

Kahler, M. y Lake, D. A. (2013). Politics in the New Hard Times: The Great Recession in Comparative Perspective. Ithaca NY: Cornell University Press.

Kaplan, S. B. (2013). Globalization and austerity politics in Latin America. Nueva York: Cambridge University Press. 
Kastner, L. (2014). “Much ado about nothing?” Transnational civil society, consumer protection and financial regulatory reform. Review of International Political Economy, 21(6), 1313-1345.

Katzenstein, P. J. (1985). Small states in world markets: Industrial policy in Europe. Ithaca, NY: Cornell University Press.

Kaufman, R. R. (2011). The political left, the export boom, and the populist temptation. En S. Levitsky y K. M. Roberts (eds.), The resurgence of the Latin American Left (pp. 93-116). Baltimore MD: The Johns Hopkins University Press.

Kingstone, P. R. (2001). Why free trade "losers" support free trade industrialists and the surprising politics of trade reform in Brazil. Comparative Political Studies, 34(9), 986-1010.

Kitschelt, H. Lange, P. et al. (1999). Continuity and change in contemporary capitalism. Cambridge: Cambridge University Press.

Korpi, W. (1983). The democratic class struggle. Londres Boston: Routledge \& Kegan Paul.

Krippner, G. R. (2012). Capitalizing on crisis: The political origins of the rise of finance. Cambridge, Mass; Londres: Harvard University Press.

Levi, M. (2000). The economic turn in comparative politics. Comparative Political Studies, 33(6-7), 822-844.

Levitsky, S. y Murillo, M. V. (2010). Variación en la fortaleza institucional. Revista de Sociología, 24, 1-242.

Levitsky, S. y Murillo, M. V. (2013). Building institutions on weak foundations. Journal of Democracy, 24(2), 93-107.

Macpherson, C. B. (1979). La teoría politica del individualismo posesivo: de Hobbes a Locke. Barcelona: Fontanella.

Madariaga, A. (2018). Variedades de capitalismo y su contribución al estudio del desarrollo en América Latina. Política y Gobierno, 25(2): 441-468.

Mair, P. (2015). Gobernando el vacio. La banalización de la democracia occidental. Barcelona: Alianza Editorial.

Mansfield, E. D. y Milner, H. V. (2012). Votes, vetoes, and the political economy of international trade agreements. Princeton NJ: Princeton University Press.

Milonakis, D. y Fine, B. (2009). From political economy to economics. Method, the social and the historical in the evolution of economic theory. Londres y Nueva York: Routledge.

Mirowski, P. y Plehwe, D. (2009). The road from Mont Pelerin: The making of the Neoliberal thought collective. Cambridge, MA: Harvard University Press.

Mirowski, P. (2013). Never let a serious crisis go to waste: How Neoliberalism survived the financial meltdown. Londres: Verso.

Montecinos, V. y Markoff, J. (2016). Economistas en las Américas. Santiago de Chile: Ediciones UDP.

Nelson, J. M. (1990). Economic crisis and policy choice: the politics of adjustment in the third world. Princeton, NJ: Princeton University Press.

North, D. C. (2006). Instituciones, cambio institucional y desempeño económico. México DF: Fondo de Cultura Económica. 
O’Donnell, G. (1978). State and alliances in Argentina, 1956-1976. Journal of Development Studies, 15(1), 3-33.

Palma, G. (1978). Dependency: A formal theory of underdevelopment or a methodology for the analysis of concrete situations of underdevelopment? World Development, 6(7-8), 881-924.

Persson, T. y Tabellini, G. E. (2002). Political economics: Explaining economic policy. Londres: MIT Press.

Polanyi, K. (2011). The economy as instituted process. En M. S. Granovetter y R. Swedberg (eds.), The sociology of economic life (pp. 22-51). Londres: Westview Press.

Pontusson, J. (1995). From comparative public policy to political economy putting political institutions in their place and taking interests seriously. Comparative Political Studies, 28(1), 117-147.

Pontusson, J. (2007). Methods in comparative political economy. Capitalisms Compared, 24, pp. 325-333.

Pribble, J. (2013). Welfare and party politics in Latin America. Nueva York: Cambridge University Press.

Rodrik, D. (2015). Economics rules: The rights and wrongs of the dismal science. Nueva York: W. W. Norton \& Company.

Rodrik, D. y Zeckhauser, R. (1988). The dilemma of government responsiveness. Journal of Policy Analysis and Management, 7(4), 601-620.

Sandel, M. (2013). Lo que el dinero no puede comprar. Barcelona: Debate.

Satz, D. (2010). Why some things should not be for sale: The moral limits of markets. Oxford, Nueva York: Oxford University Press.

Schamis, H. E. (2002). Re-forming the State: the politics of privatization in Latin America and Europe. Ann Arbor, MI: University of Michigan Press.

Schmidt, V. A. (2008). Discursive institutionalism: The explanatory power of ideas and discourse. Annual Review of Political Science, 11(1), 303-326.

Schmidt, V. A. y Thatcher, M. (2013). Resilient Liberalism in Europe's political economy. Nueva York: Cambridge University Press.

Schneider, B. R. (2004). Organizing interests and coalitions in the politics of market reform in Latin America. World Politics, 56(3), 456-479.

Schneider, B. R. (2013). Hierarchical capitalism in Latin America: Business, labor, and the challenges of equitable development. Nueva York: Cambridge University Press.

Schneider, B. R. y Karcher, S. (2010). Complementarities and continuities in the political economy of labour markets in Latin America. Socio-Economic Review, 8(4), 623-651.

Schneider, B. R. y Soskice, D. (2009). Inequality in developed countries and Latin America: Coordinated, liberal and hierarchical systems. Economy and Society, 38(1), 17-52.

Schumpeter, J. A. (1990). Historia del análisis económico. México DF: Fondo de Cultura Económica.

Sewell, W. H. (2008). The temporalities of capitalism. Socio-Economic Review, 6(3), 517-537.

Silva, E. (1996). The State and capital in Chile: Business elites, technocrats, and market economics. Boulder, CO: Westview Press. 
Smelser, N. J. y Swedberg, R. (2005). Introducing economic sociology. En N. J. Smelser y R. Swedberg (eds.), The handbook of economic sociology, $2^{\text {nd }}$ ed. (pp. 3-25). Princeton, NJ: Princeton University Press.

Smith, W. C., Luna, J. P. et al. (2014). Special section: Political economy and the future of Latin American politics. Latin American Politics and Society, 56(1), 1-33.

Stallings, B. (1992). International influence on economic policy: Debt, stabilization, and structural reform. En S. Haggard y R. R. Kaufman (eds.), The Politics of Economic Adjustment (pp. 41-88). Princeton, NJ: Princeton University Press.

Stigler, G. J. (1984). Economics: The imperial science? The Scandinavian Journal of Economics, 86(3), 301-313.

Stilwell, F. (2016). ¿Economía heterodoxa o economía política? Revista de Economía Institucional, 18(34), 283-292.

Strange, S. (1998). Mad Money. Ann Arbor, MI: University of Michigan Press.

Streeck, W. (1997). Benefitial constraints: on the economic limits of rational voluntarism. En J. R. Hollingsworth y R. Boyer (eds.), Contemporary capitalism: the embeddedness of institutions. Cambridge: Cambridge University Press.

Streeck, W. (2012). How to study contemporary capitalism? European Journal of Sociology / Archives Européennes de Sociologie, 53(01), 1-28.

Streeck, W. (2016). Comprando tiempo. La crisis pospuesta del capitalismo democrático. Buenos Aires: Katz editores.

Streeck, W. (2018). E pluribus unum? Variedades y generalidades del capitalismo. En F. González y A. Madariaga (eds.), La constitución social, politica y moral de la economía chilena (pp. 43-105). Santiago de Chile: RIL editores.

Thelen, K. (2012). Varieties of capitalism: Trajectories of liberalization and the new politics of social solidarity. Annual Review of Political Science, 15(1), 137-159.

Uzzi, B. (1997). Social structure and competition in interfirm networks: The paradox of embeddedness. Administrative Science Quarterly, 42(1), 35-67.

Wallerstein, I., Collins, R. (2013). Does capitalism have a future? Oxford y Nueva York: Oxford University Press.

Wasko, J. (2013). The IAMCR political economy section: A retrospective. The Political Economy of Communication, 1(1), 1-128.

Wehner, L. (2011). Chile's rush to free trade agreements. Revista de Ciencia Politica, 31(2), 207-226.

Weingast, B. R. y Wittman, D. A. (2006). The Oxford handbook of political economy. Nueva York: Oxford University Press.

Weyland, K. (2007). Bounded rationality and policy diffusion: Social sector reform in Latin America. Princeton, N.J: Princeton University Press.

Weyland, K. (2009). The rise of Latin America's two lefts: Insights from rentier State theory. Comparative Politics, 41(2), 145-164.

Woll, C. (2008). Firm interests: How governments shape business lobbying on global trade. Ithaca, NY: Cornell University Press. 